\title{
NASA/TM- \\ ACCELEROMETER PLACEMENT FOR THE INTERNATIONAL SPACE STATION NODE MODAL TEST
}

\author{
Michael L. Tinker * \\ Structural Dynamics and Loads Branch/ED23 \\ Structures and Dynamics Laboratory \\ NASA/Marshall Space Flight Center \\ Huntsville, AL 35812
}
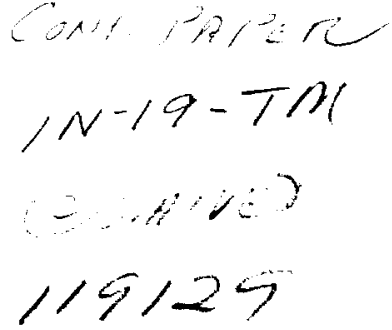

\begin{abstract}
Accelerometer location analysis for the modal survey test of the International Space Station Node is described. Three different approaches were utilized: 1. Guyan reduction, 2 . iterative Guyan reduction, and 3. the average driving point residue (ADPR) method. Both Guyan approaches worked well, but poor results were observed for the ADPR method.

Although the iterative Guyan approach appears to provide the best set of sensor locations, it is intensive computationally, becoming impractical for large initial location sets. While this is computer dependent, it appears that initial sets larger than about 1500 degrees of freedom are impractical for the interative technique.
\end{abstract}

\section{Test Configuration and Fixtures}

The modal survey test of the International Space Station Node (Fig. 1) was one of the largest known tests in regard to the volume of instrumentation required, utilizing over 400 triaxial accelerometers and more than 1200 data channels. A primary reason for the large number of accelerometers was the requirement of including numerous components interior to the Node shell (Fig. 2). Great care was taken in all phases of test planning, pre-test analysis, and conduct of the test due to the importance of the Node as the first U.S.-built component of the Space Station to be launched.

Testing was done in a large fixed-base fixture developed specifically for Space Station modules, but which can be used for any trunnion- and keel-mounted Space Shuttle payload (Ref. 1). Figure 1 shows the Node mounted in the fixture. The test fixture utilizes flexure mechanisms to simulate the Shuttle Orbiter payload constraints. These mechanisms constrain translational motion in two degrees of freedom (DOF) at each primary trunnion, and one DOF at each secondary trunnion and the keel (Fig. 2). Reference 1 provides a description of the flexure mechanisms and their development.

\section{*Aerospace Technologist, Structural Dynamics; Senior Member AIAA}

Copyright $\Theta 1998$ by the American Institute of Aeronautics and Astronautics, Inc. No copyright is asserted in the United States under Title 17, U.S. Code. The U.S. government has a royalty-free license to exercise all rights under the copyright claimed herein for Governmental purposes. All other rights are reserved by the copyright owner.

\section{Description of Approaches Used for Sensor Location Analysis}

Sensor location analysis for modal testing begins with engineering judgment supplemented by analysis to determine an initial set of measurement locations. Of course, locations that are not accessible, as well as rotational DOF, can be immediately removed from consideration. This initial set is much smaller than the full finite element model, but considerably larger than a practical final set of locations. The initial set of locations can partly be determined by visual inspection of the structure's geometry and mode shapes to determine critical regions of the structure for instrumentation. Critical regions will have well-defined motion in one or more of the target mode shapes, or provide paths by which loads are transmitted into the structure. Kinetic energy and mass-to-stiffness ratio calculations can also be used to help locate or verify critical locations.

Once the analyst has determined the character of the mode shapes and identified several important areas to be instrumented, the initial set of sensor locations includes these areas and also provides generally good coverage of the structure to define the mode shapes. A lot of conservatism can be utilized in the choice of the initial set to make sure that all possible regions of interest are covered. For example, initial sets of size greater than 2000 DOF may be reasonable for large complex structures. The next step in the process is to use analytical techniques to reduce the large initial set to a realistic size, which can be on the order of 200-400 locations or more for very large modal tests.

Several analytical techniques are commonly used for determining measurement locations, including kinetic energy sorting, iterative Guyan reduction (Refs. 2-3), and effective independence (Ref. 4). Reference 3 provides a good overview of these first three methods. Other techniques that have been investigated include use of flexibility shapes and genetic algorithms, as discussed in Refs. 5-7. Listed below are several techniques that were determined to be of interest for selection of measurement locations for the Node modal test.

\section{Kinetic Energy Sorting}

As described in Ref. 8, kinetic energy sorting involves an examination of each DOF's contribution of kinetic energy to each mode shape. By summing the energy over all the modes for each DOF, those coordinates having the greatest contribution or most energy can be indentified and retained in a candidate set. 
Reference 8 indicates that a problem with this approach lies in its inability to recognize when many DOF have approximately equal kinetic energies, and that it cannot retain one such coordinate without retaining them all.

\section{Guyan Reduction}

Standard or noniterative Guyan reduction (Ref. 2 ) involves examining each DOF in the full model to determine which DOF have the largest diagonal mass to diagonal stiffness ratio. That is, the locations on the structure where inertia forces are large compared to elastic forces are to be retained. A sorting procedure can be used for finding the $\mathrm{N}$ degrees of freedom with the largest diagonal $M / K$ ratio in descending order.

The result of this process is that a reduced model is generated that accurately preserves the dynamic characteristics of the structure at the lower frequencies (Refs. 3-4). If some higher-order modes are of interest for model correlation, then a larger set should be retained, a different technique should be used, or the results should be modified using engineering judgment.

\section{Iterative Guyan Reduction}

In this approach (Refs. 3-4), the ratio of diagonal mass to diagonal stiffness is again examined, but the DOF with the smallest ratio is removed. The mass and stiffness matrices are reduced, and the process is repeated. This procedure is continued until the desired model size and accuracy are achieved. The advantage of this iterative process is that the effects of each removed DOF are distributed to the remaining DOF, providing greater accuracy than the standard or non-iterative approach (Ref. 3).

\section{Average Driving Point Residue (ADPR) Method}

This approach is utilized in commercial software for modal testing and model correlation (Ref. 9). As described in Ref. 3, it uses an average magnitude or amplitude of mode shapes. Degrees of freedom with the highest average driving point residue, or highest weighted average modal magnitude, could make up a measurement set. A sorting procedure can be used to list these DOF in descending order.

\section{Previous Comparative Siudies}

As stated in Ref. 6, "...no one method stands out as the clear choice. Methods which perform well in one instance may give completely unacceptable results in another." However, the authors of Ref. 6 go on to point out that the commonly used methods such as iterative Guyan reduction, kinetic energy sorting, and effective independence typically give reasonable results. Results of some comparative studies of several techniques are described in this section.

The comparative study in Ref. 6 describes selection of sensor locations for the Pegasus launch vehicle constrained at attach locations to the carrier aircraft. The full model had approximately $30,000 \mathrm{DOF}$, and the initial candidate measurement set consisted of 150 locations and 450 DOF. Several commonly-used methods (kinetic energy sorting, iterative Guyan reduction, and effective independence) were evaluated for the problem and compared to results using flexibility shapes. The methods were compared for a reduction of the measurement set to 150 DOF (300 coordinates eliminated), and 24 target modes to $50 \mathrm{~Hz}$ were selected. For that particular application, kinetic energy sorting, mass weighted effective independence, and iterative Guyan reduction performed best.

A second comparative study is described in Ref. 8, where kinetic energy sorting, iterative Guyan reduction, effective independence, and genetic algorithm methods were cvaluated for four structural models. The test structures evaluated were a general-purpose spacecraft model, 10-bay space truss, avionics box, and satellite model. Size of the full models ranged from 360 DOF for the 10-bay truss to about 22,000 DOF for the satellite model. The initial set size varied from 168 to 576 DOF, and the final accelerometer set size was typically on the order of 30-75 DOF. Results consistently showed good performance of all four methods, but the genetic algorithm seeded with results of the other algorithms did best followed by iterative Guyan reduction.

Reference 3 compares the iterative Guyan reduction and ADPR methods for a cantilever beam, free-free $\mathrm{H}$-frame, 2-D truss pinned at one end, and a free-free plate. Generally, the iterative Guyan procedure gave the best results, particularly for constrained structures with no rigid body modes. For free-free structures or those with one or more rigid body modes, the ADPR approach seemed to work better, but iterative Guyan also did an adequate job. Both methods are easily implemented.

In conclusion of comparative studies, iterative Guyan reduction fares very well generally for different boundary conditions, though kinetic energy sorting and mass-weighted effective independence also did quite well. The ADPR method appears to work well for free-free structures, or those with rigid body modes. Newer approaches such as genetic algorithms show great potential, but do not appear to be as easily implemented as the the more commonly used methods, and apparently must be seeded with results of the other algorithms. Based on these findings, Guyan reduction was given considerable attention in the accelerometer location analysis for the Space Station Node, as described in the remainder of the paper.

\section{Application of Methods to the International Space Station Node}

The general approach taken for accelerometer placement for the Node external shell included the following steps: 1 . Begin with a fairly large set based mainly on visual inspection of the structure geometry and analytical mode shapes, but also based on kinetic energy sorting, 2. Add locations known to be paths by which loads are transmitted to the structure, and other locations that appear to be of interest, such as trunnion and keel support structures, shell reinforcing rings, and end cones, 3. Run iterative Guyan reduction, beginning with the set described in 2 ., to reduce the number of locations for the Node external shell to about 190,4 . Use standard non-iterative Guyan reduction and ADPR reduction to also obtain candidate sets of measurement coordinates, 5. Run eigenvalue analyses for the reduced models and a reference Craig-Bampton model (Ref. 6) or full model, and form cross-orthogonality of the resulting modes normalized with respect to the reduced mass matrix. 
Results of frequency comparisons and crossorthogonality calculations were used as the figures-of-merit or standards by which each candidate set and reduction technique was evaluated. In Tables 1 and 2 , the crossorthogonality values are shown for two initial sets, one with nearly 500 external shell locations and 1500 DOF and the second with approximately 800 locations and 2400 DOF. It was found that the iterative Guyan approach provided measurement sets that compared very well with the reference model. Table 3 shows the cross-orthogonality values for a set reduced to 195 locations and 577 DOF. Comparison with Tables 1 and 2 verifies the good performance of the iterative Guyan approach. Poor results in all cases for modes 19-22 were found to be due to improper constraints for some internal connections in the Node finite element model. When the constraints were corrected, good orthogonality and frequency comparisons were obtained for modes 19-22.

The ADPR method did not perform well for the Node structure, as seen in Table 4. Possible this is because the Node test was a fixed-boundary configuration. Results in Ref. 3 suggest that the ADPR approach works better for free-free test configurations.

It was also found in this study that the kinetic energy sorting method as a stand-alone sensor location procedure did not work well. It was discovered that the method provided locations on the structure that are heavy and stiff, and not a good distribution of desirable measurement points.

\section{Summary and Conclusions}

This paper describes results of accelerometer placement analysis for the International Space Station Node fixed-base modal survey test. It was found that the iterative Guyan reduction method performed very well, yielding a measurement set with good frequency and crossorthogonality comparisons to the reference model. However, the iterative method was computationally intensive, requiring long run times (about 4 hours wallclock time for the initial 1500 DOF set, and 2 weeks for the initial 2400 DOF set). Although the run times are dependent on computer platform and workload, it is clear that the iterative approach becomes impractical for initial candidate sets larger than about 1500 DOF.

Standard non-iterative Guyan reduction also provided a good measurement set, but the ADPR technique gave poor results for the Node structure in a constrained configuration.

\section{References}

1. Tinker, M. L., "Modal Vibration Test Facilities and Methods for Space Station Modules," AIAA Paper 951295, 1995.

2. Guyan, R.J., "Reduction of Stiffness and Mass Matrices", AlAA Journal, Vol. 3, No. 2, p. 380.

3. Penny, J.E.T, Friswell, M.I., and Garvey, S.D., "The Automatic Choice of Measurement Locations for Dynamic Testing", AJAA Joumal, Vol. 32, No. 2, 1994, pp. 407-414.

4. Kammer, D.C., "Sensor Placement for On-Orbit Modal Identification and Correlation of Large Space Structures", Joumal of Guidance. Control. and Dynamics, Vol. 14, No. 2, pp. 251-259.

5. Flanigan, C.C., and Botos, C.D., "Automated Selection of Accelerometer Locations for Modal Survey Tests", Proceedings of the 10th International Modal Analysis Conference, San Diego, CA, Feb. 3-7, 1992, pp. 1205-1208.

6. Stabb, M., and Blelloch, P., "Application of Flexibility Shapes to Sensor Selection", 13th International Modal Analysis Conference, Detroit, MI, Feb. 12-15, 1995, pp. 1255-1262.

7. Stabb, M.C., and Blelloch, P.A., "A Genetic Algorithm for Optimally Selecting Accelerometer Locations", Proceedings of the 13th International Modal Analysis Conference, Detroit, MI, Feb. 12-15, 1995, pp. 1530-1534.

8. Flanigan, C.C., and Stabb, M.C., Jr., "Comparison of Automated Methods for Optimum Accelerometer Selection", 13th International Modal Analysis Conference, Detroit, MI, Feb. 12-15, 1995.

9. LMS International, "Large-Scale Modal Testing of a Space Frame Structure--From Pretest Analysis fo FEA Model Validation," Sound and Vibration, March 1991, pp. 6-16.

10. Craig, R. R., Jr., and Bampton, M. C. C., "Coupling of Substructures for Dynamic Analysis", AlAA Journal, Vol. 6, No. 7, July 1968, pp. 1313-1319.

\section{Acknowledgments}

Steven Woletz of Boeing Company in Huntsville, Alabama determined initial candidate accelerometer locations for the Node exterior, as well as the final set used in the modal test. Bobby Evars, also of the Boeing Company in Huntsville, determined sensor locations for the Node interior components. 


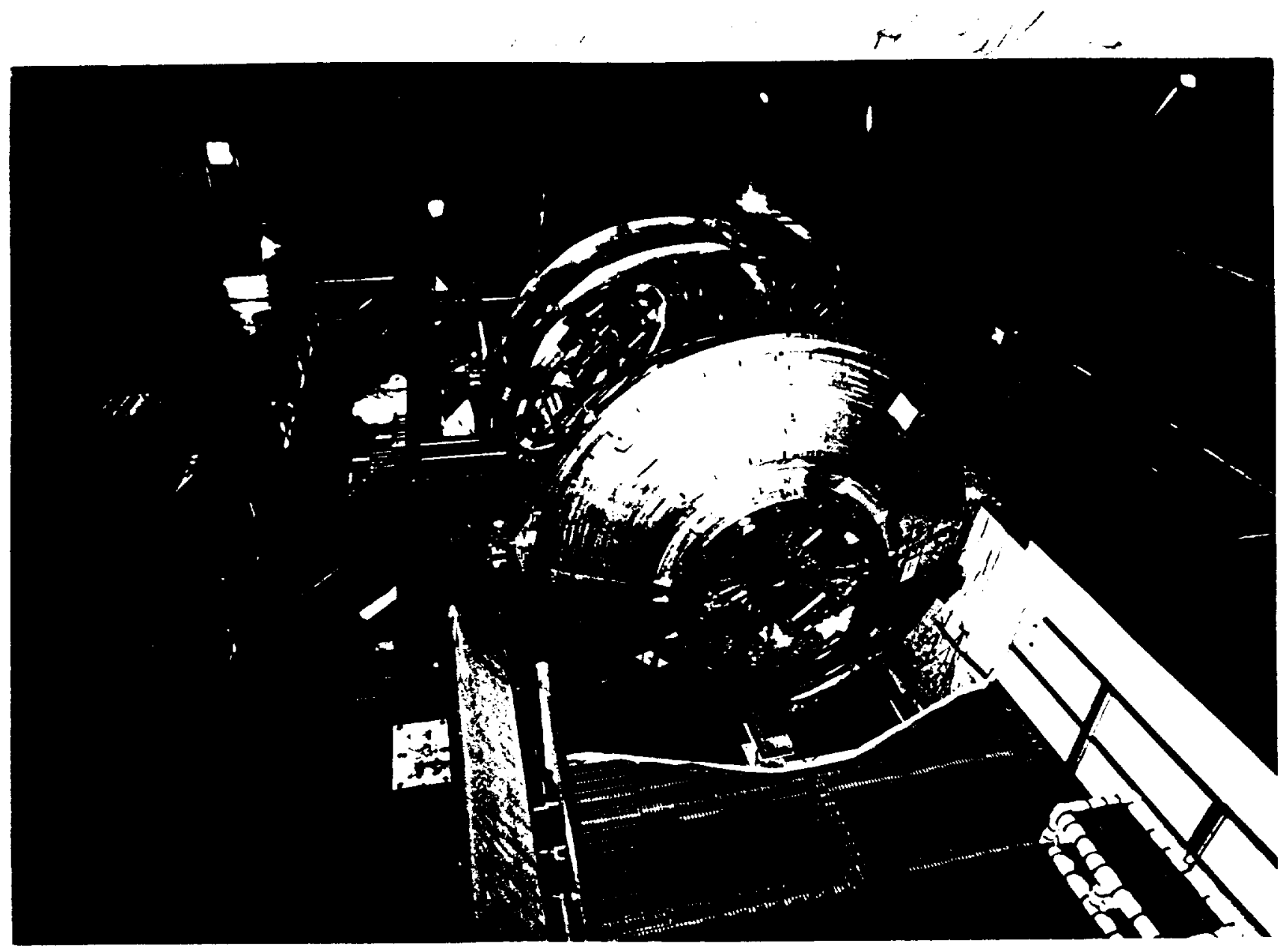

Figure 1. International Space Station Node in Modal Test Configuration 

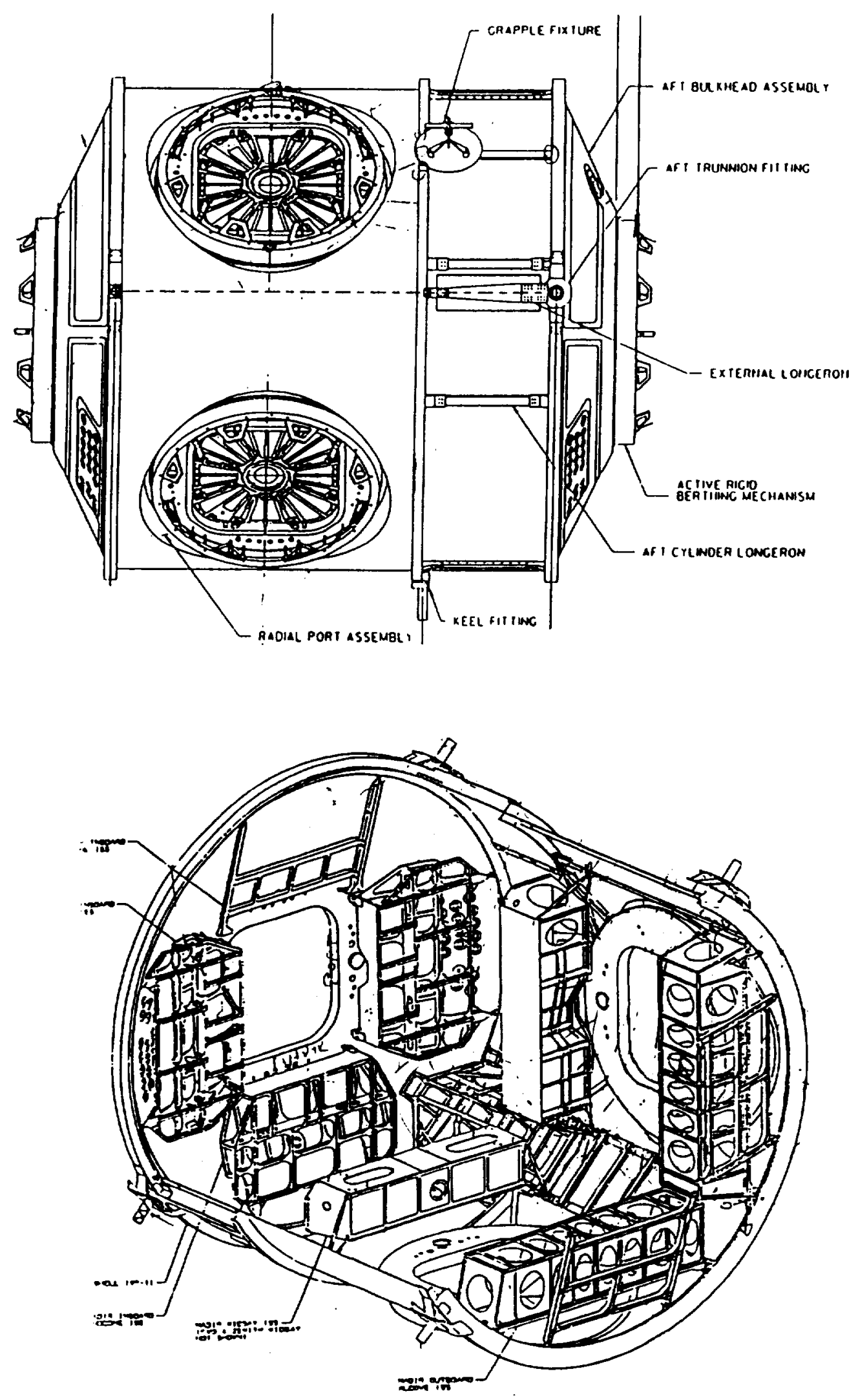

Figure 2. Space Station Node External Shell and Internal Structure 
Table 1. Constrained Frequency and Mode Comparisons for 1500 DOF Initial Set and Full Model
Table 2. Frequency and Mode Shape Comparisons for 2400 DOF Initial Set and Full Model

\begin{tabular}{|c|c|c|c|c|}
\hline & Full & & Reduced & Correl. \\
\hline $\begin{array}{l}1 \\
2 \\
3 \\
4 \\
5 \\
6 \\
7 \\
8 \\
9 \\
10 \\
11 \\
12 \\
13 \\
14 \\
15 \\
16 \\
17 \\
18 \\
19 \\
20 \\
21 \\
22 \\
23 \\
24 \\
25 \\
26 \\
27 \\
28\end{array}$ & $\begin{array}{r}7.3152 \\
10.7081 \\
11.1486 \\
14.6754 \\
17.8832 \\
18.1015 \\
18.7835 \\
21.0240 \\
21.1056 \\
21.3428 \\
22.3180 \\
22.5698 \\
22.6876 \\
23.2005 \\
24.0261 \\
24.2233 \\
24.933 \\
25.5163 \\
25.7490 \\
25.8087 \\
25.8489 \\
25.9833 \\
26.5815 \\
26.7932 \\
27.3517 \\
28.0258 \\
28.1892 \\
28.2009 \\
28.3107 \\
28.3453\end{array}$ & $\begin{array}{r}1 \\
2 \\
3 \\
1 \\
5 \\
6 \\
7 \\
8 \\
9 \\
10 \\
11 \\
12 \\
13 \\
14 \\
15 \\
16 \\
17 \\
18 \\
19 \\
21 \\
22 \\
18 \\
19 \\
20 \\
21 \\
22 \\
23\end{array}$ & $\begin{array}{r}7.3161 \\
10.7113 \\
11.4549 \\
11.6805 \\
17.8851 \\
18.1042 \\
18.7913 \\
21.1234 \\
21.2139 \\
21.4625 \\
22.3595 \\
22.6420 \\
22.7260 \\
23.2457 \\
24.0306 \\
21.2530 \\
24.9727 \\
25.7124 \\
26.5827 \\
27.5732 \\
28.1236 \\
25.7121 \\
26.5827 \\
26.8195 \\
27.5732 \\
28.1236 \\
28.2791 \\
28.3112 \\
28.3923 \\
28.5274\end{array}$ & $\begin{array}{r}-1.00000 \\
1.00000 \\
-1.00000 \\
1.00000 \\
-1.00000 \\
0.99999 \\
-0.99999 \\
-0.99908 \\
-0.99919 \\
-0.99983 \\
-0.99666 \\
0.98907 \\
-0.99202 \\
-0.99932 \\
-0.99970 \\
0.99909 \\
0.99507 \\
-0.98312 \\
-0.63313 \\
-0.13050 \\
0.24650 \\
0.75599 \\
-0.99812 \\
-0.99941 \\
-0.99831 \\
-0.98411 \\
-0.81537 \\
0.78309 \\
-0.99533 \\
0.99176\end{array}$ \\
\hline
\end{tabular}

Table 3. Comparison of Model Reduced Using Iterative Guyan Reduction to 577 DOF and Full Model

\begin{tabular}{|c|c|c|c|c|}
\hline & Full & & Reduced & Correl. \\
\hline $\begin{array}{l}1 \\
2 \\
3 \\
4 \\
5 \\
6 \\
7 \\
8 \\
9 \\
10 \\
11 \\
12 \\
13 \\
16 \\
15 \\
16 \\
17 \\
18 \\
19 \\
20 \\
21 \\
22 \\
23 \\
24 \\
25 \\
26 \\
27\end{array}$ & $\begin{array}{r}7.2196 \\
10.6819 \\
11.4300 \\
14.6456 \\
17.7610 \\
17.9643 \\
18.7479 \\
21.0198 \\
21.1021 \\
21.3405 \\
22.0126 \\
22.3859 \\
22.5785 \\
22.9062 \\
23.6384 \\
23.8784 \\
24.6093 \\
25.3845 \\
25.7470 \\
25.8082 \\
25.8482 \\
25.9622 \\
26.5742 \\
26.7992 \\
27.3403 \\
28.0028 \\
28.9151 \\
28.1937 \\
28.3062\end{array}$ & $\begin{array}{r}1 \\
2 \\
3 \\
1 \\
5 \\
6 \\
7 \\
8 \\
9 \\
10 \\
11 \\
12 \\
13 \\
14 \\
15 \\
16 \\
17 \\
18 \\
19 \\
21 \\
22 \\
19 \\
19 \\
20 \\
21 \\
22 \\
24 \\
23 \\
25\end{array}$ & $\begin{array}{r}7.2205 \\
10.6850 \\
11.4363 \\
14.6507 \\
17.7628 \\
17.9670 \\
18.7561 \\
21.1186 \\
21.2103 \\
21.4601 \\
22.0415 \\
22.4125 \\
22.6385 \\
22.9511 \\
23.6431 \\
23.9065 \\
24.6257 \\
25.5880 \\
26.5738 \\
27.4616 \\
28.1058 \\
25.52890 \\
26.5738 \\
26.8268 \\
27.4616 \\
38.1058 \\
38.2998 \\
38.2781 \\
28.3876 \\
28.4217\end{array}$ & - \\
\hline
\end{tabular}

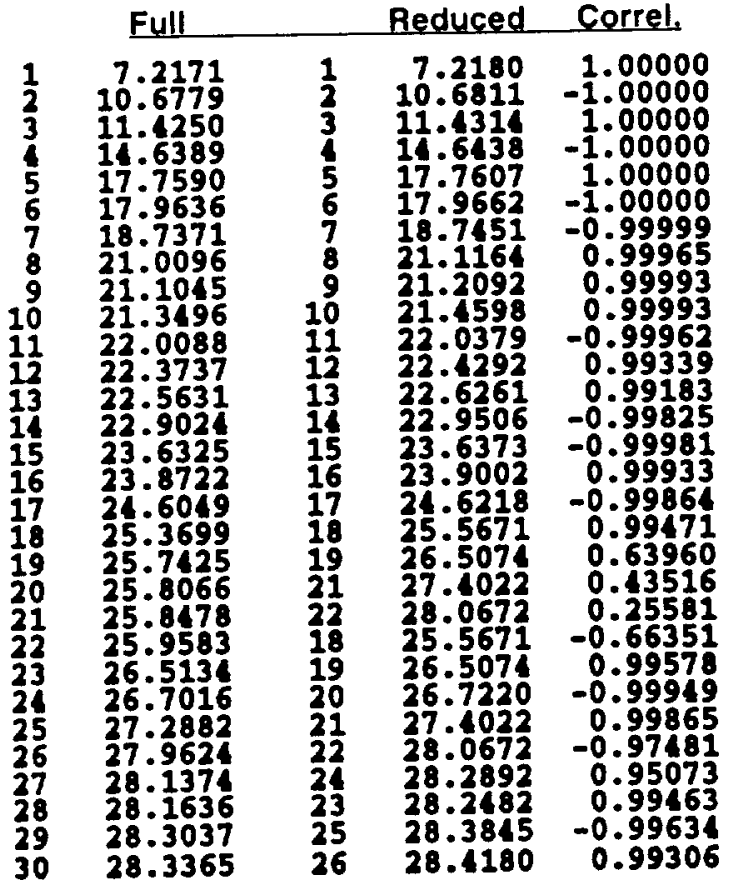

Table 4. Results for Model Reduced Using ADPR Method in Comparison to Full Model

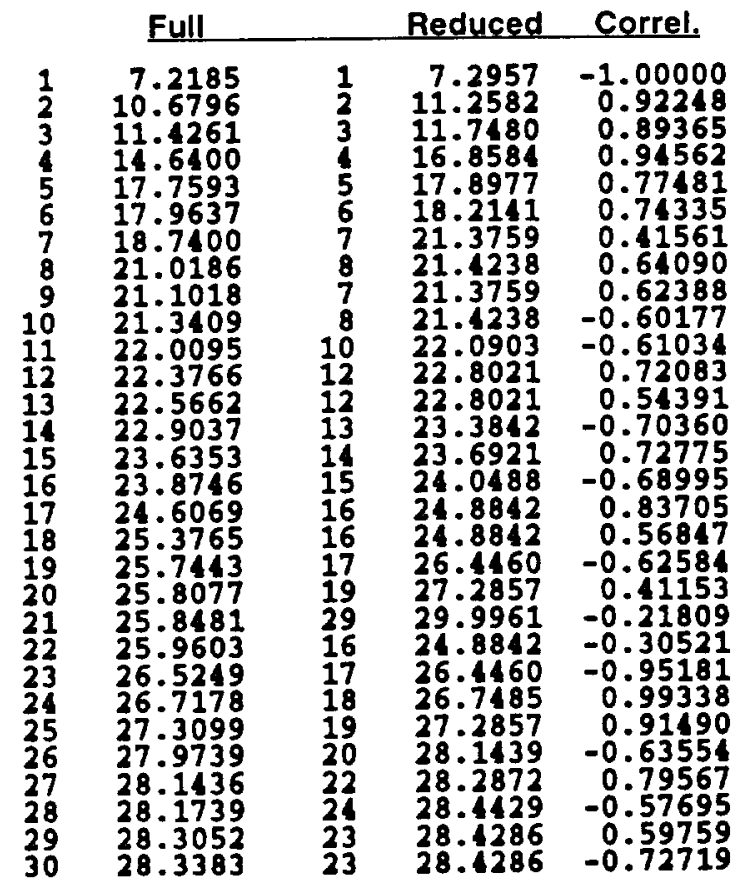

